\title{
Recent developments in biologic therapies for the treatment of patients with systemic lupus erythematosus
}

\author{
Pedro L. Carreira ${ }^{1}$ and David A. Isenberg ${ }^{2}$
}

\begin{abstract}
SLE has a complex pathogenesis, and multiple therapeutic targets have been discovered in recent years. In spite of belimumab being approved by the US Food and Drug Administration and the widespread use of rituximab, there have been many failed attempts to treat SLE successfully using biologic agents. In this review, we consider newer biologic approaches that might offer the hope of improving the outcome of SLE patients. These include the fully humanized anti-CD20 mAbs, PEGylated anti-CD40L, IFN $\alpha$ inhibitors, rigerimod and immune complexes blockade.
\end{abstract}

Key words: lupus, biologic, anti-CD20, anti-CD40L, dapirolizumab, ofatumumab, obinutuzumab, rigerimod, interferon, $\mathrm{FC} \gamma \mathrm{R}$

\section{Rheumatology key messages}

- There is still considerable morbidity with conventional immunosuppression in SLE patients.

- Successful biologic use in SLE is a decade behind that for rheumatoid and psoriatic arthritis.

- A number of new promising biologic therapies for SLE are in clinical trials.

\section{Introduction}

The use of biologic therapies in the treatment of patients with SLE is, arguably, at least a decade behind their use in RA, PsA and AS. The need for more successful biologic or other new therapies remains important as it is clear we have reached the limit of what can be achieved with conventional immunosuppression [1]. SLE patients have $\sim 90 \%$ survival rate at 10 years [2] with considerable morbidity, which remains very unsatisfactory for a disease that often develops before age 30 .

Some encouragement is taken from the approval of belimumab by the US Food and Drug Administration (and more recently the National Institute for Health and Clinical Excellence) and the widespread use of rituximab (in spite of two trials that did not meet their endpoints). Neither drug alone will be a panacea for SLE, although

\footnotetext{
${ }^{1}$ Autoimmune Diseases Unit, Internal Medicine 7.2 Department, Hospital Curry Cabral, Centro Hospitalar Lisboa Central, Lisbon, Portugal and ${ }^{2}$ Department of Rheumatology, University College London, London, UK

Submitted 9 June 2017; revised version accepted 12 February 2018

Correspondence to: David A. Isenberg, Room 424 The Rayne Building, Centre For Rheumatology, The Division of Medicine, University College London, 5 University Street, London WC1E 6JF, UK.

E-mail: d.isenberg@ucl.ac.uk
}

interestingly attempts to combine those drugs are now being pursued. In this mini-review we will focus on several new or modified approaches that we believe offer the best hope of improving the outcome of SLE patients. Our choice of new approaches is subjective, based on our reading of the recent literature.

\section{Pathogenesis}

The pathogenesis of SLE involves genetic and epigenetic factors, environmental triggers and immunological abnormalities. These abnormalities include defective apoptosis and loss of tolerance; inadequate development of dendritic cells; defective function of regulatory $T$ cells and $B$ cells; defective $B$ and $T$ cells apoptosis and defective signalling pathways. Fig. 1 shows the link between these factors and the sites of action of relevant therapeutic agents.

\section{CD20 blockade}

B cells play an essential role in the development of SLE. Blocking $\mathrm{B}$ cells with rituximab, a chimeric $\mathrm{mAb}$ against antigen CD20, is well established in SLE. Because it is a chimeric antibody, can cause allergic responses in $\sim 10 \%$ of SLE patients. Fully humanized mAbs anti-CD20 have been developed including ofatumumab and obinutuzumab. 
FIG. 1 Simplified diagram of SLE pathogenesis and site of action of therapeutic agents

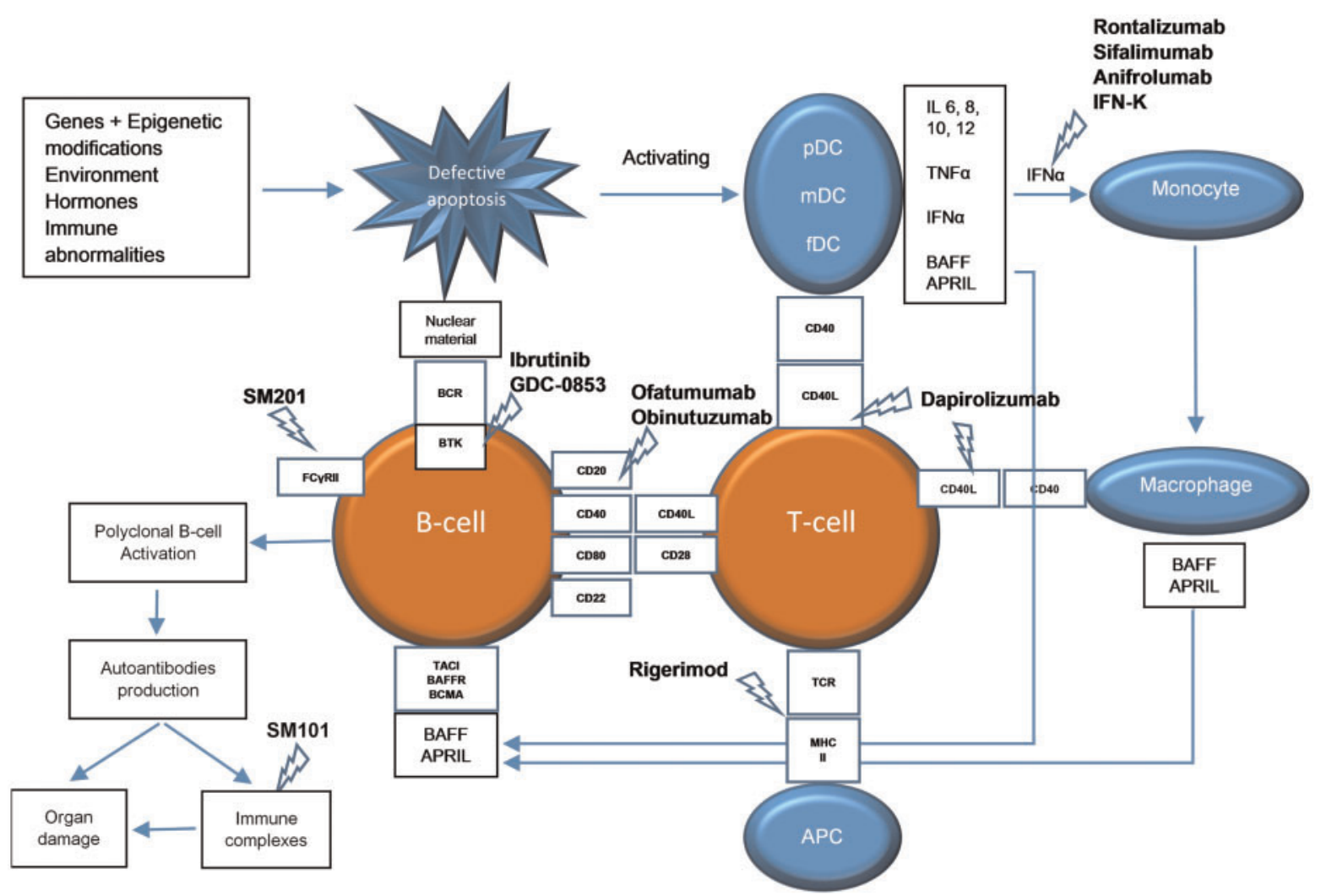

Defective apoptosis results in production of uncleared nuclear material leading to dendritic-cells (DC) activation and BCR stimulation. DC will produce a number of cytokines, which will result in B-cell activation (BAFF and APRIL) but also differentiation of monocytes to macrophages (IFN $\alpha$ ) that will present self-antigens to $T$ and $B$ cells but also produce cytocines. The activation of B cells needs a co-stimulatory signal between T-cell receptor (TCR) and MHC in antigen presenting cells (APC) but also CD40: CD40L binding with macrophage and dendritic cells. B-cells activation, differentiation, and proliferation leads to autoantibody production. Immune complex formation and tissue deposition results in organ damage. pDC: plasmacytoid dendritic cells; mDC: myeloid dendritic cells; fDC: follicular dendritic cells; BCR: B-cell receptor; BTK: Bruton's tyrosine kinase; BAFF: B cell activating factor; APRIL: a proliferation inducing ligand; APC: antigen presenting cells.

Ofatumumab is a mAb IgG1 anti-CD20 approved for chronic lymphocytic leukaemia. Experience of its use in SLE is restricted to a small number of cases. For example, an SLE patient whose previous flares had responded to rituximab, but became allergic to it, received three infusions of ofatumumab and achieved an SLEDAl decrease from 15 to 2, a reduction of anti-dsDNA antibodies levels $>90 \%$ and C3 normalization [3]. An SLE patient with autoimmune haemolytic anaemia refractory to rituximab, achieved B-cell depletion after ofatumumab, with remission of autoimmune haemolytic anaemia and a SLEDAI of 0 [4]. Four patients with LN achieved proteinuria and anti-dsDNA reduction after ofatumumab [5]. Formal clinical trials in SLE are awaited.

Obinutuzumab, a glycol-engineered mAb anti-CD20, is being used in the treatment of non-Hodgkin's lymphoma. An in vitro study comparing rituximab to obinutuzumab in SLE demonstrated the latter to be a more efficient B-cell depletor [6]. A 52-week, phase II trial studying safety and efficacy in LN is currently recruiting (NCT02550652). Its primary outcome is complete renal response.

\section{B-cell intracellular signalling blockade-Bruton's tyrosine kinase}

Bruton's tyrosine kinase (BTK) is a component of B-cell receptor signalling, involved in regulating cell proliferation and survival. Its blockade results in B-cell apoptosis. B-cells overexpressing BTK in mice led to anti-dsDNA antibody production and SLE-resembling organ involvement. Many inhibitors of BTK are in development, including ibrutinib and GDC-0853.

Ibrutinib is a tyrosine kinase selective and irreversible inhibitor. It binds to BTK causing B-cell apoptosis. Preclinical trials showed ibrutinib reduced levels of autoantibodies (anti-nucleosome, anti-histone and anti-ssDNA but not anti-dsDNA) and renal disease [7]. However, no current clinical trial is ongoing in SLE. 
GDC-0853 is another BTK inhibitor [8]. A phase II trial in SLE is ongoing (NCT02908100), to evaluate the safety and efficacy in patients with moderate-to-severely active SLE.

\section{T cell co-stimulation blockade}

B-cell immune stimulation follows interaction with $\mathrm{T}$ cells and antigen-presenting cells via co-stimulatory signals, notably CD40/40 L, CD28, cytotoxic T-lymphocyte antigen 4, and CD80/CD86.

Rigerimod is a 21-mer linear peptide derived from the small nuclear RNP U1-70 K, whose mechanism of action seems to be due to chaperone-mediated autophagy [9]. By reducing the stability of $\mathrm{MHC}$ molecules that present antigens to $\mathrm{T}$ cells, it blocks antigen presentation to autoreactive $T$ cells, which in turn blocks $B$-cells maturation.

A phase II trial involved 20 patients with moderately active SLE who received three s.c. injections. Significant improvement in the SLEDAl score was reported with the $200 \mu \mathrm{g} /$ dose [10]. A phase Illb trial showed a significant reduction of disease activity [11]. One hundred and forty-nine patients were randomized to receive rigerimod or a placebo every 2 or 4 weeks. Patients with SLEDAI-2K $\geqslant 6$ who received rigerimod $200 \mu \mathrm{g}$ every 4 weeks achieved a statistically higher Systemic Lupus Erythematosus Responder Index (SRI)-4 response at week 12 (67.6 vs $41.5 \%, P<0.025)$ and week 24 (84.2 vs $45.8 \%, P<0.025)$. A phase III trial is underway, whose primary outcome is SLEDAl-2 K reduction $\geqslant 4$ (NCT02504645).

CD40 ligand (CD40L) is a protein expressed on activated $\mathrm{T}$ cells and a member of the TNF family. Its binding to CD40 on antigen-presenting cells and B cells induces co-stimulation and promotes B-cell maturation. AntiCD40L mAbs block co-stimulation in experimental models. A phase II trial in 28 patients with proliferative LN showed significant reduction in circulating levels of anti-dsDNA antibodies and increased C3 levels, but was associated with thromboembolic events [12]. Another phase II trial of a different anti-CD40L mAb had no major adverse events (AE), however, efficacy was not proven [13]. The thromboembolic events were evidently caused by the functional Fc region of anti-CD40L, which triggers platelet aggregation by interacting with platelet Fc $\gamma$ RIIA receptor. In dapirolizumab the Fc portion has been changed to a high molecular polyethylene glycol without loss of efficacy [14]. A 32-week, phase IB trial showed safety and tolerability of intravenous dapirolizumab in SLE patients. Clinical response was evident by both SRI and BILAG-based Combined Lupus Assessment (BICLA) assessments [15]. A 24-week phase II trial, followed by observational period to evaluate efficacy and safety on moderately to severely active SLE is recruiting (NCT02804763). The primary outcome is the BICLA response rate at 24 weeks.

\section{Interferon blockade}

IFNs are a family of glycoproteins that consist of type I IFN (IFN-I) (including 12 isoforms of IFN $\alpha$ and one of IFN $\beta$ ) and type II IFN (includes only IFN $\gamma$ ). IFN-I binds to the type I
IFN receptor (IFNR), IFN $\gamma$ binds to another receptor. IFN activates multiple signalling pathways, especially Janus kinase. IFN dysregulated activity and signalling is associated with autoimmune disease development. Rarely, when IFN $\alpha$ has been used, for example, in hepatitis C and cryoglobulinaemic vasculitis, autoimmune conditions, including SLE, have been reported to develop [16, 17]. IFN-I levels are higher in SLE and recent studies have shown a link between IFN $\alpha$ levels and disease activity. In Ifnar1 gene knockout mice, reduced disease activity is seen.

Rontalizumab is a humanized IgG1 mAb against IFN $\alpha$. A phase I trial proved safe but showed no clinical benefit in those with a high IFN signature [18]. A phase II trial evaluated efficacy and safety of rontalizumab in 159 patients with moderate to severe SLE. Patients were randomized to receive rontalizumab $750 \mathrm{mg}$ or placebo every 4 weeks plus standard of care (SOC) (part 1) and subsequently, $300 \mathrm{mg}$ rontalizumab or placebo every 2 weeks (part 2). The results did not confirm rontalizumab's general superiority, however paradoxically, in the low IFN signature group SRI response rates were superior in 31\% $(P=0.0285)$ and the SELENA-SLEDAI flare index rate was reduced [19]. However, no further trial is ongoing.

Sifalimumab is a fully human $\operatorname{lgG} 1 \kappa \mathrm{mAb}$ that binds to most subtypes of IFN $\alpha$ and neutralizes it. Two phase I trials showed safety and IFN signature reduction in a dose-dependent manner. A 52-week phase Ilb trial followed by a 22-week safety follow-up, enrolled 431 patients randomized to receive placebo or sifalimumab (200, 600 or $1200 \mathrm{mg}$ ) every 28 days in addition to SOC. In all sifalimumab groups, the response rates were significantly higher than in the placebo group, however, only the group with the high IFN signature patients achieved significant improvement in SRI-4. Cutaneous Lupus Erythematosus Disease area and Severity Index (CLASI) as well as joint counts showed significant improvement. No differences in lowering anti-dsDNA antibodies or normalizing C3/C4 levels were observed. Herpes zoster was the principal $A E$ as expected by IFN suppression [20]. Currently no phase III trial is ongoing.

Unlike the other IFN blockers, directed against IFN, anifrolumab is a fully human $\lg \mathrm{G} 1 \kappa \mathrm{mAb}$ that blocks subunit 1 of the IFNR and consequently both IFN $\alpha$ and IFN $\beta$. A phase I trial showed safety and sustained IFN signature reduction in SSc patients. In a 52-week phase Ilb lupus trial, 305 patients were randomized to receive placebo, anifrolumab $300 \mathrm{mg}$ or $1000 \mathrm{mg}$ every 4 weeks until week 48 as well as SOC [21]. Active LN or neuropsychiatric SLE were excluded. The primary efficacy end point was a combination of the SR- 4 at weeks 24 and 52 with a sustained reduction in oral corticosteroids from weeks 12 to 24. At both weeks 24 and 52, the response was achieved in a significantly higher number of patients receiving both dosages of anifrolumab $(300 \mathrm{mg}$, $P=0.014$ and $1000 \mathrm{mg}, P=0.063)$. When evaluating the sub-groups by IFN signature levels, those with high levels of IFN achieved significantly better results compared with placebo at both 24 and 52 weeks. Significant 


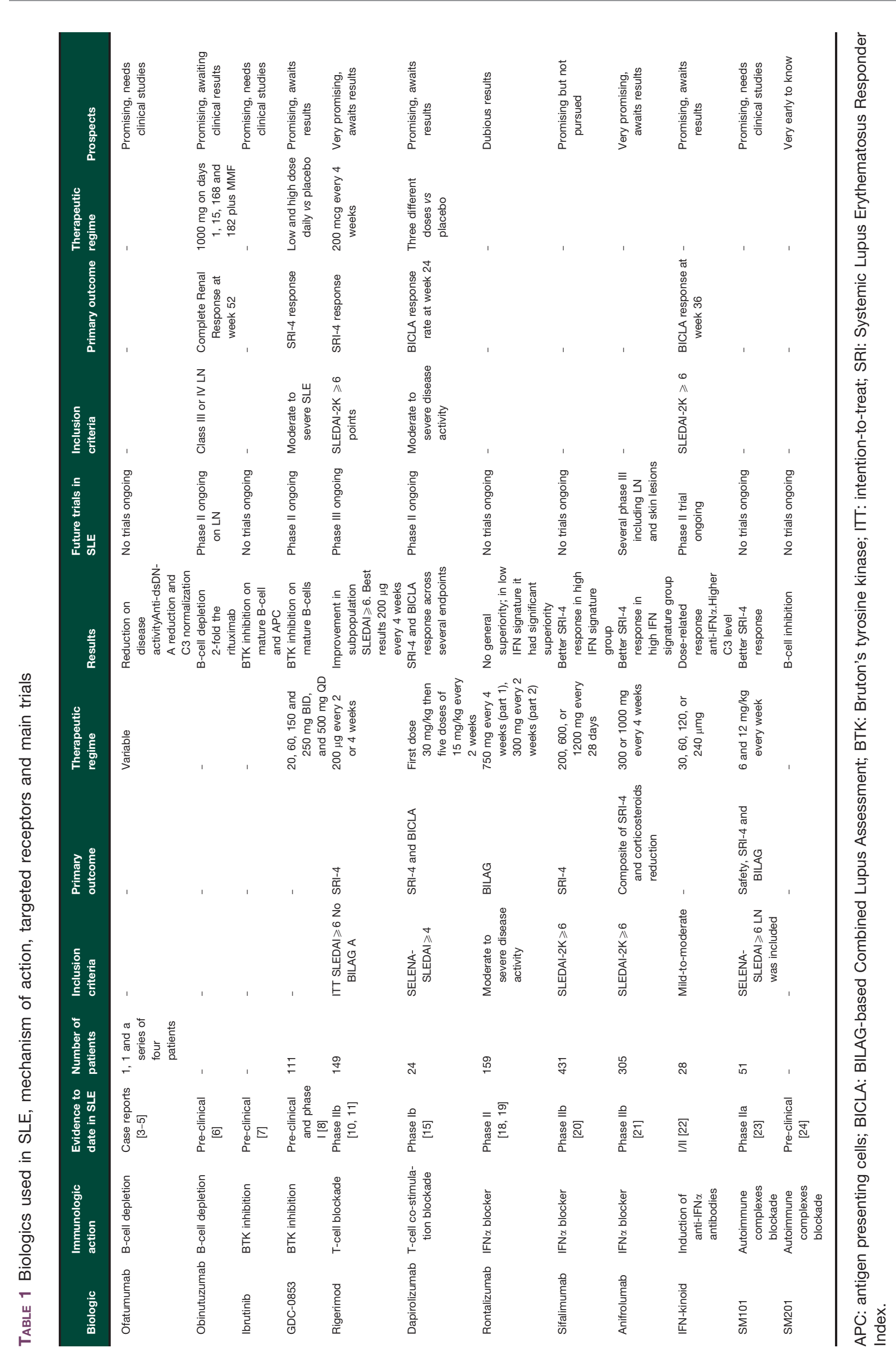


improvement was also achieved using BICLA, BILAG 2004; CLASI; joint counts and other variables. Influenza and herpes zoster infections were the most frequent $\mathrm{AE}$. Further studies are ongoing in active SLE patients, including in LN (NCT02547922).

IFN- $\alpha$-kinoid (IFN-K) is another option in blocking IFN $\alpha$. IFN-K is a vaccine composed of IFN $\alpha 2 b$ coupled to a carrier protein. It acts by inducing antibody production against all IFN $\alpha$ subtypes. A phase I/II trial of 28 patients with mild-to-moderate SLE showed a dose-related antiIFN $\alpha$ response and improvement of C3 levels [22]. A phase II trial is ongoing to elicit IFN signature reduction and efficacy in SLE (NCT02665364).

\section{Blocking immune complexes}

Fc $\gamma$ receptors $(F c \gamma R)$ are transmembrane proteins that recognize the $\mathrm{Fc}$ region of $\mathrm{lgG}$. The binding of immune complexes (ICs) and Fc $\gamma \mathrm{R}$ initiates intracellular signalling, which results in an autoimmune response. Most of the $\mathrm{Fc} \gamma \mathrm{R}$ molecules act as activating receptors and only Fc $\gamma$ RIIB is an inhibitory receptor. Both types of receptors are expressed on the same cells. Negative signalling by Fc $\gamma$ RIIB is mainly important for the regulation of activated $B$ cells. SLE patients have a lower expression of Fc $\gamma$ RIIB.

SM101 is an extracellular version of the human Fc $\gamma$ RIIB. It binds to IC in SLE and blocks the Fc $\gamma$-mediated signal. Fc $\gamma$ RIIB was chosen as a therapeutic target because of its limited human polymorphism and lack of immunogenicity. In a 24-week phase Ila trial, 51 SLE patients were randomized to receive SM101 or placebo weekly for 4 weeks. The primary outcome was safety and the secondary outcomes included SLEDAI, BILAG, physician global assessment, global response and renal parameters. No serious $\mathrm{AE}$ were reported. The SRI-4 response was twice as high in the SM101 group and in LN results was even better [23]. The results seem promising but phase III trials are needed.

Rather than binding to IC, binding to the receptor itself can produce an inhibitory response. SM201 is an antiFc $\gamma R$ IIIB mAb, it binds to Fc $\gamma$ RIIB but allows the binding between IC and the Fc $\gamma$ RIIB. A pre-clinical study showed that SM201 had a synergic action with IC resulting in a better inhibition of B cells [24]. It also seems to be restricted to activated $B$ cells, allowing a functional memory response. Clinical trials are needed to understand if it's a valid therapeutic.

A resume of ongoing trials relevant to the approaches discussed in this review are shown in Table 1.

\section{Conclusions}

Biologic treatment of lupus has seen many false dawns. The relative successes of belimumab, rituximab, and atacicept (not discussed here) have been counterbalanced by the failures of many others including abatacept, tabalumab, blisibimod, and epratuzumab. But a number of new approaches, including fully humanized anti-CD20, PEGylated anti-CD40L, IFN $\alpha$ inhibitors and rigerimod, offer new hope that before too long we will have a range of biologic options to offer our SLE patients that matches the choices we have for our RA and PSA patients.

Other approaches, not discussed here, such as blocking the Janus kinase/signal transducers and activators of transcription-pathway, II-6 and nuclear factor kappa-lightchain-enhancer of activated $B$ cells might also prove of value.

Funding: No specific funding was received from any bodies in the public, commercial or not-for-profit sectors to carry out the work described in this manuscript.

Disclosure statement: D.A.I. has received honoraria from Merck Serono, Eli Lilly, XTLBio, Anthera, Celgene and UCB Pharma; these honoraria are passed on to a local arthritis charity. The other author has declared no conflicts of interest. The authors acknowledge the support of the Biomedical Research Centre (BRC) Award to University College Hospital and University College London.

\section{References}

1 Croca SC, Rodrigues T, Isenberg DA. Assessment of a lupus nephritis cohort over a 30-year period. Rheumatology 2011;50:1424-30.

2 Mak A, Cheung MW, Chiew HJ, Liu Y, Ho RC. Global trend of survival and damage of systemic lupus erythematosus: meta-analysis and meta-regression of observational studies from the 1950s to 2000s. Semin Arthritis Rheum 2012;41:830-9.

3 Thornton CC, Ambrose N, loannou Y. Ofatumumab: a novel treatment for severe systemic lupus erythematosus. Rheumatology 2015;54:559-60.

4 Karageorgas T, Zomas A, Kazakou P et al. Successful treatment of life-threatening autoimmune haemolytic anaemia with ofatumumab in a patient with systemic lupus erythematosus. Rheumatology 2016;55:2085-7.

5 Haarhaus ML, Svenungsson E, Gunnarsson I. Ofatumumab treatment in lupus nephritis patients. Clin Kidney J 2016;9:552-5.

6 Reddy V, Klein C, Isenberg DA et al. Obinutuzumab induces superior B-cell cytotoxicity to rituximab in rheumatoid arthritis and systemic lupus erythematosus patient samples. Rheumatology 2017;56:1227-37.

7 Hutcheson J, Vanarsa K, Bashmakov A et al. Modulating proximal cell signaling by targeting Btk ameliorates humoral autoimmunity and end-organ disease in murine lupus. Arthritis Res Ther 2012;14:R243.

8 Katsumoto TWH, Kotwal S, Murray E et al. Safety, pharmacokinetics, and biomarker profile from phase 1 clinical trials of healthy volunteers treated with GDC-0853, a highly selective reversible oral bruton's tyrosine kinase (BTK) inhibitor. In: Arthritis Rheumatol 2016;68 (Suppl 10): Abstract 2622, p. 3544-3545. WILEY, American College of Rheumatology, 2016 ACR/ARHP Annual Meeting Washington, DC. http://acrabstracts.org/wp-content/ uploads/2016/10/ACR-2016-Abstracts-PDF.pdf. 
9 Macri C, Wang F, Tasset I et al. Modulation of deregulated chaperone-mediated autophagy by a phosphopeptide. Autophagy 2015;11:472-86.

10 Muller S, Monneaux F, Schall N et al. Spliceosomal peptide P140 for immunotherapy of systemic lupus erythematosus: results of an early phase II clinical trial. Arthritis Rheum 2008;58:3873-83.

11 Zimmer R, Scherbarth HR, Rillo OL, Gomez-Reino JJ, Muller S. Lupuzor/P140 peptide in patients with systemic lupus erythematosus: a randomised, double-blind, placebo-controlled phase Ilb clinical trial. Ann Rheum Dis 2013;72:1830-5.

12 Boumpas DT, Furie R, Manzi S et al. A short course of BG9588 (anti-CD40 ligand antibody) improves serologic activity and decreases hematuria in patients with proliferative lupus glomerulonephritis. Arthritis Rheum 2003;48:719-27.

13 Kalunian KC, Davis JC Jr, Merrill JT et al. Treatment of systemic lupus erythematosus by inhibition of $T$ cell costimulation with anti-CD154: a randomized, doubleblind, placebo-controlled trial. Arthritis Rheum 2002;46:3251-8.

14 Tocoian A, Buchan P, Kirby $\mathrm{H}$ et al. First-in-human trial of the safety, pharmacokinetics and immunogenicity of a PEGylated anti-CD40L antibody fragment (CDP7657) in healthy individuals and patients with systemic lupus erythematosus. Lupus 2015;24:1045-56.

15 Chamberlain CUM, Soranson J, Watling M et al. Repeated administration of dapirolizumab pegol (DZP) appears safe and well tolerated in patients with systemic lupus erythematosus (SLE) and is accompanied by an improvement in disease activity: results from a phase 1 study. In: Arthritis Rheumatol 2015;67 (Suppl 10): Abstract 3222, p. 3864-3865. WILEY, American College of Rheumatology, 2015 ACR/ARHP Annual Meeting - San Francisco, CA. http://acrabstracts.org/wp-content/ uploads/2017/10/abstracts-acr-2015.pdf.

16 Hardy GA, Sieg S, Rodriguez B et al. Interferon- $\alpha$ is the primary plasma type-I IFN in HIV-1 infection and correlates with immune activation and disease markers. PLoS One 2013;8:e56527.

17 Niewold TB, Swedler WI. Systemic lupus erythematosus arising during interferon-alpha therapy for cryoglobulinemic vasculitis associated with hepatitis C. Clin Rheumatol 2005;24:178-81.

18 McBride JM, Jiang J, Abbas AR et al. Safety and pharmacodynamics of rontalizumab in patients with systemic lupus erythematosus: results of a phase I, placebocontrolled, double-blind, dose-escalation study. Arthritis Rheum 2012;64:3666-76.

19 Kalunian KC, Merrill JT, Maciuca R et al. A Phase II study of the efficacy and safety of rontalizumab (rhuMAb interferon- $\alpha$ ) in patients with systemic lupus erythematosus (ROSE). Ann Rheum Dis 2016;75:196-202.

20 Khamashta M, Merrill JT, Werth VP et al. Sifalimumab, an anti-interferon- $\alpha$ monoclonal antibody, in moderate to severe systemic lupus erythematosus: a randomised, double-blind, placebo-controlled study. Ann Rheum Dis 2016;75:1909-16.

21 Furie R, Khamashta M, Merrill JT et al. Anifrolumab, an anti-interferon- $\alpha$ receptor monoclonal antibody, in moderate-to-severe systemic lupus erythematosus. Arthritis Rheumatol 2017;69:376-86.

22 Lauwerys BR, Hachulla E, Spertini F et al. Down-regulation of interferon signature in systemic lupus erythematosus patients by active immunization with interferon $\alpha$-kinoid. Arthritis Rheum 2013;65:447-56.

23 Tillmanns S, Kolligs C, D'Cruz DP et al. SM101, a novel recombinant, soluble, human Fc gamma Ilb receptor, in the treatment of systemic lupus erythematosus: results of a double-blind, placebo-controlled multicenter study. Arthritis Rheum 2014;66:S1238.

24 Rieth N, Carle A, Müller MA et al. Characterization of SM201, an anti-hFc $\gamma$ RIIB antibody not interfering with ligand binding that mediates immune complex dependent inhibition of B cells. Immunol Lett 2014;160:145-50. 